\title{
Top-down Modulation of Motor Priming by Belief about Animacy
}

\author{
ROMAN LIEPELT ${ }^{1}, \&$ MARCEL BRASS ${ }^{1,2}$ \\ 1 Max Planck Institute for Human Cognitive and Brain Sciences, Department of Psychology, \\ Leipzig, Germany \\ 2 Ghent University, Department of Experimental Psychology and Ghent Institute for \\ Functional and Metabolic Imaging, \\ Ghent, Belgium \\ In Press, Experimental Psychology
}

Words: 3589

Running head: Animacy and Motor Priming

\section{Corresponding author:}

Roman Liepelt

Max Planck Institute for Human Cognitive and Brain Sciences

Department of Psychology

Stephanstr. 1A

04103 Leipzig

Germany

Phone: +49 341 - 9940 - 2279

E-mail: liepelt@cbs.mpg.de 


\begin{abstract}
There is recent evidence that we directly map observed actions of other agents onto our own motor repertoire, referred to as direct matching (Iacoboni et al., 1999). This was shown when we are actively engaged in joint action with others' (Sebanz et al. 2003) and also when observing irrelevant movements while executing congruent or incongruent movements (Brass et al., 2000). However, an open question is whether direct matching in humans is limited to the perception of intentional agents. Recent research provides contradictory evidence with respect to the question whether the direct matching system has a biological bias possibly emerging from perceptual differences of the stimulus display. In the present study all participants performed a motor priming task observing the identical animation showing finger lifting movements of a hand in a leather glove. Before running the experiment we presented either a human hand or a wooden analogue hand wearing the leather glove. We found a motor priming effect both, for human and wooden hands. However, motor priming was larger when participants believed that they interacted with a human hand than when they believed to interact with a wooden hand. The stronger motor priming effect for the biological agent suggests that the 'direct matching system' is tuned to represent actions of animate agents.
\end{abstract}

Keywords: motor priming; direct matching; biological agents 


\section{INTRODUCTION}

It is now widely acknowledged that observed behaviour is automatically mapped onto the observer's motor repertoire (Iacoboni, Woods, Brass, Bekkering, Mazziotta, \& Rizzolatti, 1999). This ability to motorically simulate other people's behaviour is believed to provide the foundation for social interactions (Decety \& Jackson, 2004) and action understanding (Gallese \& Goldman, 1998).

Behaviourally, direct matching of others' actions was shown with motor priming paradigms (Brass \& Heyes, 2005 and Blakemore \& Frith, 2005). In such paradigms, participants have to carry out simple actions in response to a symbolic stimulus while observing congruent or incongruent actions. In a widely used experimental setup participants have to respond to a symbolic number stimulus ("1" or "2") while observing a task-irrelevant interfering finger lifting movement of another person. It was demonstrated that the observation of the finger movement activates a corresponding motor response in the observer which can lead to facilitation if the movement is congruent and to interference if it is incongruent (Brass, Bekkering, Wohlschläger, \& Prinz, 2000; Bertenthal, Longo, \& Kosobud, 2006).

\section{Biological tuning of direct matching?}

It is an open question whether direct matching in humans is limited to the perception of intentional agents. A biological tuning is predicted by theories assuming that direct matching originates from sensori-motor learning (Brass \& Heyes, 2005; Keysers \& Perrett, 2004). Behavioural studies have found such a bias for human action as compared to robot actions (Kilner, Paulignan, \& Blakemore, 2003; Press, Bird, Flach, \& Heyes, 2005; Tsai and Brass, 2007) and so do some neuroimaging studies (Perani, Fazio, Borghese, Tettamanti, Ferrari, Decety, \& Gilardi, 2001; Tai, Scherfler, Brooks, Sawamoto, Castiello, 2004). Evidence for a human-specific direct-matching system is, however, not unambiguous. A recent neuroimaging study showed contrasting evidence when comparing human and robotic actions (Gazzola, 
Rizzolatti, Wicker, \& Keysers, 2007). Also new behavioural work (Jansson, Wilson, Williams, \& Mon-Williams, 2007; Stanley, Gowen, \& Miall, 2007) showed comparable effects elicited by simple moving dots when the dot stimulus followed a biologically plausible or implausible velocity profile. When participants were informed that they observe dot stimuli that represent pre-recorded human movements they found an interference effect but the effect was absent when the dot motion was described as computer generated. In line with the latter findings Liepelt, von Cramon, \& Brass (2008) showed differential automatic imitation effects for human finger movements depending on whether the observer attributed the movement as intentionally produced or not. The aim of the present study was to test whether the direct matching system is prone to top-down influence or not. This was tested by manipulating participant's belief regarding the animacy of the stimuli. All participants performed the same motor priming task observing an animation of a leather hand. Before running the experiment, two groups of subjects were presented two different static hand models (human/wooden). We showed one group of subjects a real human hand wearing a leather glove and the other group a wooden analogue model of a hand wearing the leather glove (see photographs of the human and wooden hand wearing the glove in Fig. 1A and B for illustration). We told participants that they will see the respective model also on the screen during the experiment. Both groups saw the identical stimulus on the screen showing an ambigous hand stimulus in a leather glove (see Fig. 1C).

We predicted that, if the direct matching system is tuned to the perception of intentional agents, one should find increased motor priming effects for the leather hand animation when participants believed that it was produced by a human. If, however, the direct matching system represent others' actions independently of the nature of the agent no group differences should be found. 


\section{Method}

\section{Participants}

A group of 20 undergraduate students (9 male, mean age: 23,6) participated in this experiment. Ten were given pre-experimental experience with a human leather hand and ten were given experience with a wooden leather hand. Participants were randomly assigned to both groups. All were right handed, had normal or corrected-to-normal vision, and were naive with regard to the hypotheses of the experiment. Participants were paid $7 €$ for participation.

\section{Apparatus and stimuli}

Stimuli were presented on a 17-inch color monitor that was connected to a Pentium I PC. Experiments were carried out using ERTS software (Experimental Runtime System; Beringer, 2000). The sequence of hand movements consisted of two pictures of a right hand positioned in the same perspective as the hand of the participant with which the action was executed (see Fig. 1C). The presented sequence produced realistic finger movements. As stimuli, we presented a hand in a leather glove. We tried to create a relatively ambiguous stimulus that would work for both, human and wooden hand models. Actually we used a real hand in the glove. To introduce the belief manipulation we used a real human hand (Human) and a wooden analogue model of a hand, both in a leather glove. At a viewing distance of $80 \mathrm{~cm}$, the hand on the screen subtended a visual angle of $9.57^{\circ} \times 10.27^{\circ}$. The movie consisted of two frames only. One starting frame which showed the hand in a resting position and a movement frame that showed either the index or the middle finger lifted. Simultaneously with the movement frame the numbers were presented between the index and the middle finger of the videotaped hand. The number subtended a visual angle of $0.72^{\circ} \times 0.36^{\circ}$.

Please insert Figure 1 about here 


\section{Procedure and Design}

An adopted version of the paradigm developed by Brass et al. (2000) was used in the present experiment. Participants had to lift either the index or the middle finger of their right hand in response to a number (1: index, 2: middle). The number was displayed together with a photo of a right hand from a first person's perspective on a computer screen, the same orientation as participant's response hand. The number always appeared at the same position, located between the index finger and the middle finger of the observed hand. As the number appeared, either the index or the middle finger of the observed hand was lifted either congruently or incongruently to the required response indicated by the number (see Fig. 1C). In congruent trials, the observed and the required response finger were identical. In incongruent trials, the observed and the required response finger differed. Participants were instructed to respond to the numbers irrespective of the observed finger lifting movements.

Each trial began with a frame lasting for $800 \mathrm{~ms}$ showing a resting hand. The second frame showed the same hand with one of the fingers completely lifted for $1915 \mathrm{~ms}$. At the same time as the movement was presented, additionally a digit appeared between the index and the middle finger of the observed hand. A tonal feedback that informed participants about the correct use of the response board was given for $50 \mathrm{~ms}$ followed by a blank screen of $2100 \mathrm{~ms}$. Thus each trial lasted 4865 ms (see Fig. 2).

The belief manipulation was realized in the following way. Before running the experiment, we presented either a static hand of a real human wearing a leather glove (Human group) or a wooden analogue model of a hand wearing the leather glove (Wooden group). Both groups were given the identical verbal instruction, that they would see short clips of the respective hand model on the screen during the experiment. This statement was embedded within the overall instructions. We presented the identical leather hand movement to both groups (between-group design) to avoid low-level stimulus confounds. In each group the movement 
was presented for two blocks each consisting of 120 trials, separated by a short break. Participant performed 240 trials in total.

Please insert Figure 2 about here (Trial sequence)

\section{Rating data}

To investigate how participants of both groups interpreted the observed hand stimulus, we acquired additional data. 1) After having performed the experiment, participants had to complete a questionnaire, in which they were asked to indicate the extent to which (they thought) the static hand model (human/wooden) and the stimulus hand presented on the screen during the experiment were matched. 2) To give an indication of the perceived intentionality of the observed movement participants were asked to rate how intentional they perceived the observed movement to be. Participants responded to both questions using a 5point scale ranging from not at all to fully.

\section{Results}

\section{Data analysis}

In all experiments prior to statistical analyses, all trials in which responses were incorrect or slower than 2000 ms were excluded from statistical Reaction Time (RT) analyses. This resulted in the elimination of $2.1 \%$ of trials from the data set. One participant was excluded from the human group, due to high error rates of more than 15 percent. RTs for all conditions are presented in Fig. 3a.

\section{RT analysis}

We used a 2 x 2-factorial design including the 2-level between-subject factor Group (human, wooden) as well as the 2-level factor Congruency (congruent, incongruent) as a within-subject variable (see Fig. 3a). The same analysis was conducted for accuracy data (see Fig. 3b). 
In the present experiment, we observed a main effect of Group, $F(1,17)=8.76, \mathrm{MSe}=$ 2615.08, $p<.05$, partial $\eta^{2}=.34$, participants in the human group were slower than in the wooden group. Furthermore, we found a main effect of Congruency, $F(1,17)=201.16$, MSe $=267.61, p<.001$, partial $\eta^{2}=.92$, due to increased RTs in incongruent compared to congruent conditions. Most importantly, a significant interaction between Group $x$ Congruency was observed, $F(1,17)=10.05, \mathrm{MSe}=267,61, p<.05$, partial $\eta^{2}=.37$, indicating a significantly larger motor priming effect in the human-hand model group as compared to the wooden-hand model group.

Please insert Figures 3a and 3b (RTs and percent errors) about here

Post hoc test indicated that in the human-hand model group, attributing the movement as triggered by a human agent led to a significant congruency effect of $\mathbf{9 2} \mathbf{~ m s ,} t(8)=-10.40, p<$ .001 .

In the wooden-hand model group attributing the movement as triggered by a non-human agent, also lead to a significant congruency effect of $\mathbf{5 9} \mathbf{~ m s ,} t(9)=-9.48, p<.001$. The motor priming effect in the human-hand model group was $34 \mathrm{~ms}$ larger as compared to the wooden-hand model group (see Fig. 4).

Please insert Figure 4 (Effects sizes for different conditions) about here

To rule out the possibility that a group difference might have produced the observed interaction we computed the same analysis using ratio measures adjusting for the mean reaction time difference in both groups. This was done by calculating the mean value of congruent and incongruent RTs per participant. Afterwards, we calculated the mean of these values per group, which provides a mean RT level per group. To correct for these RT 
differences, we built ratios by dividing raw data by the mean RT level per group. Then we performed the identical analyses as for raw RT data. This analysis showed a main effect of Congruency, $F(1,17)=201.04, \mathrm{MSe}=.001, p<.001$, partial $\eta^{2}=.92$, due to increased ratios in incongruent compared to congruent conditions. Importantly, we still observed a significant interaction between Group x Congruency, when accounting for possible group differences, $F(1,17)=5.70, \mathrm{MSe}=.001, p<.05$, partial $\eta^{2}=.25$, ruling out the possibility that the differential interference effect between groups was driven by mean RT differences.

\section{Error analysis}

For errors, we observed no main effect of Group, $(F<1)$, but a main effect of Congruency, $F(1,17)=23.12, \mathrm{MSe}==2.96, p<.001$, partial $\eta^{2}=.58$, due to an increased error rate in incongruent compared to congruent conditions in both groups. Furthermore, we observed no significant interaction between Group x Congruency, $(F<1)$, indicating no differences in motor priming between the human-hand model and the wooden-hand model group indicating no evidence for a speed-accuracy trade-off (SAT) as an explanation for the observed RT effects (see Fig. 3b).

\section{Rating data}

To determine participants beliefs about the hand presented on the screen, participants were asked to indicate the extent to which the model's hand and the video hand were matched. All participants made clear that they believed the stimulus hand to be the same hand as the model's hand presented at the beginning of the experiment by showing relatively high scores (average score $=4$ out of maximally 5 ) in matching ratings.

One might argue that animacy didn't make a difference for motor priming, but just the degree of match between the model hand (human/wooden) and the stimulus hand. Non-parametric analyses of the matching ratings indicated 1) that the degree of match between the model (human/wooden) and the stimulus presented on the screen were not perceived differently 
between the human-hand model group and the wooden-hand model group $(z=-.88$, twotailed Mann-Whitney U test, $p>$.38). 2) The ratings on perceived intentionality, showed a numerical trend that the movements on the screen in the human hand-model group were perceived also as more intentional as compared to the wooden hand-model group. This difference, however, missed statistical significance $(z=-1.64$, two-tailed Mann-Whitney U test, $p<.12$.). This is in line with the idea that the larger motor priming effect in the human animate condition is driven by the perceived intentionality of the movement.

\section{DISCUSSION}

The aim of the present study was to investigate the fundamental question whether the direct matching system is prone to top-down influence or not. This question was tested under perceptually identical task conditions using a belief manipulation about the animacy of an observed action. In the present study participants performed a motor priming task (Brass et al., 2000) with an animation of a moving leather hand. Before running the experiment, we presented either a human model wearing a leather glove or a wooden hand analogue wearing the leather glove. We replicated previous findings showing an automatic tendency to imitate (Brass et al., 2000; Stürmer, Aschersleben, \& Prinz, 2000) by measuring the motor priming effect produced by a task-irrelevant finger lifting movement on an actually executed movement. A basic motor priming effect was present regardless of whether participants believed that the movement was executed by a biological or a non-biological agent. However, importantly motor priming effects were larger when participants believed to interact with a human hand rather than a wooden hand. Because we used the identical stimuli for both, the human hand-model group and the wooden hand-model group, the present findings can not be explained by perceptual differences of the stimuli. These findings are in line with results showing a top-down modulation of motor priming effects (e.g. Stanley et al., 2007; Longo and Bertenthal, 2009). 
One might, however argue that the larger motor priming effect in the "real" hand movie is not due to the difference in animacy, but just to the degree of match between the static hand model presented before the experiment and the stimulus presented during the experiment. We think that this alternative explanation is rather unlikely. First, during the experimental testing the stimuli were animated while in the demonstration phase we showed an unanimated hand. Furthermore, participants rated the match between the stimuli presented in the instruction phase and the experimental phase equally high in both conditions.

Interestingly, the magnitude of the motor priming effect in the human condition of the present experiment seems larger than that observed in previous studies using a similar paradigm (Bertenthal et al., 2006; Brass et al., 2000). However, it is very difficult to compare the absolute motor priming effect across studies. First, the timing is quite different in different studies and this might have an impact on the size of the interference effect. Secondly, the perspective from which the model's hand is observed also differs between studies. Previous experiments presented stimuli from the third person's perspective while the present study uses the first persons perspective. From an ideomotor theory point of view (James, 1890; Prinz, 1997) one could argue that priming effects should be larger for the first person perspective because of the higher dimensional overlap of the observed and executed action (Kornblum, Hasbroucq, \& Osman, 1990).

In order to rule out the possibility that the higher mean reaction time in the human hand condition led to a relative increase of the interference effect we computed the relative interference effect. However, also for this ratio measure the larger interference effect in the human hand condition remained reliable. To conclude, the present findings strongly suggest that the belief about animacy modulates motor priming in a top-down manner.

\section{Intention Priming rather than Motor Priming}

This assumption is also in accordance with other recent approaches to investigate the effect of observed biological motion. Using a joint action paradigm (Sebanz et al. 2003), Tsai \& Brass 
(2007) found evidence for a joint Simon effect in a social context in which participants coacted with a computer-animated human hand. Importantly, they demonstrate that this social Simon effect was biologically tuned and occurs only when participants coact with human conspecifics. The joint Simon effect completely disappeared when participants interacted with a wooden hand analogue. However, as outlined above in contrast to the Tsai and Brass (2007) findings the motor priming effect in the present study was smaller but still present when participants believed that the observed movement was produced by a non-biological agent. This difference might provide important insights for differential mechanisms underlying joint action and automatic imitation effects. While joint action effects may be mainly driven by the belief and the knowledge about a coactor, automatic imitation effects seem to be a combined effect of belief (pure imitation effect) and a spatial compatibility component (Bertenthal et al., 2006). In line with this interpretation Tsai and colleagues (Tsai, Kuo, Hung, Tzeng, 2008) recently demonstrated joint Simon effects by simply telling participants that they interacted with another person sitting in a different room. This issue needs however further testing. The present results also relate to recent findings showing a modulation of motor priming by biological possible vs. impossible movements (Longo, Kosobud, \& Bertenthal, 2008). Longo and colleagues observed an equivalent motor priming effect when participants observed biomechanically possible and impossible movements. Only when they explicitly pointed their subjects to the fact that the impossible movement was in fact unrealistic, they found a modulation of the motor priming effect. All these manipulations might have a common underlying cause, namely manipulating the conviction about the intentional nature of the observed movement. This would be in line with the assumption that at least a portion of the motor priming effect is driven by the attribution of intention rather than by the movement itself (Liepelt et al., 2008).

At first glance the finding of a smaller motor priming effect in the wooden hand group as compared to the human hand group seem to be in contrast with previous findings showing that 
people attribute social roles and intentions even to simple moving geometric shapes (Heider and Simmel, 1944). The present findings, however seem to suggest that what matters with respect to the attribution of animacy and intentions to a certain stimulus is not only what people see, but what they believe to see is animate or not.

\section{Animacy and Motor Priming}

But how do beliefs about animacy of the observed agent influence motor priming? There are two potential interpretations for this top-down influence on motor priming. On the one hand one can argue that beliefs about animacy modulate the attention people direct to the stimuli (attention hypothesis). On the other hand, one can argue that a kind of gating mechanism controls access to the mirror system (gating hypothesis). Longo and Bertenthal (2009) recently argued in favour of the attention hypothesis. In their experiment they presented a virtual hand or a videotaped real hand. Both the virtual hand and the real hand produced a substantial motor priming effect. However, when participants where explicitly briefed that the hands were computer animated the motor priming effect was reduced for the virtual hand but not for the real hand. Longo and Bertenthal argued that the instruction raised the knowledge about the artificiality of the hand from subsidiary into focal awareness and therefore the virtual hand was perceived as virtual, leading to a smaller motor priming effect. However, in the present experiment the stimuli could be interpreted as biological or as non-biological stimuli. In fact the interpretation as biological stimuli was even more plausible. Nevertheless, a belief manipulation led to an attenuation of the motor priming effect. In this sense, our findings are more in accordance with a gating hypothesis rather then an attention hypothesis. When participants believe an observed movement is produced by a biological agent they show the classical motor priming effect. However, when they believe the movement stems from a non-intentional agent the movement does not gain privileged access to the mirror system. However, whether the belief manipulation is successful or not depends on the stimuli. When observing a virtual hand or a hand in a glove, the belief manipulation is quite plausible. 
However, when observing a real hand the manipulation is not very convincing. It is crucial to note, that these manipulations might operate on an implicit level.

\section{Conclusions}

The present findings provide further evidence for a top-down influence of beliefs on the direct matching system. Motor priming strongly depends on the interpretation of the observed behaviour even in a situation where the stimuli are physically identical. Furthermore, we propose a gating hypothesis of motor priming, suggesting that only behaviour perceived as intentional gains privileged access to the mirror system. 


\section{REFERENCES}

Beringer, J. (2000). Experimental Runtime System. BeriSoft Cooperation, Frankfurt am Main (1987-2000).

Bertenthal, B. I., Longo, M. R., \& Kosobud, A. (2006). Imitative response tendencies following observation of intransitive actions. Journal of Experimental Psychology: Human Perception and Performance, 32(2), 210-225.

Blakemore, S. J. \& Frith, C. (2005). The role of motor contagion in the prediction of action, Neuropsychologia, 43, 260-267.

Brass, M., Bekkering, H., Wohlschlager, A., \& Prinz, W. (2000). Compatibility between observed and executed finger movements: comparing symbolic, spatial, and imitative cues. Brain Cognition, 44(2), 124-143.

Brass, M. \& Heyes, C. M. 2005. Imitation: is cognitive neuroscience solving the correspondence problem? Trends in Cognitive Science, 9, 489-495.

Decety, J., \& Jackson, P. L. (2004). The functional architecture of human empathy. Behavioral and Cognitive Neuroscience Reviews, 3(2), 71-100.

Gallese, V. and Goldman, A. (1998) Mirror neurons and the simulation theory of mindreading. Trends in Cognitive Sciences, 12, 493-501.

Gazzola, V., Rizzolatti, G., Wicker, B., \& Keysers, C. (2007). The anthropomorphic brain: The mirror neuron system responds to human and robotic actions, Neuroimage 35, 16741684.

Heider, F. and Simmel, M. (1944). An experimental study of apparent behavior. American Journal of Psychology, 57, 243-259.

Iacoboni, M., Woods, R. P., Brass, M., Bekkering, H., Mazziotta, J. C., \& Rizzolatti, G. (1999). Cortical mechanisms of human imitation. Science, 286(5449), 2526-2528.

James,W. (Ed.). (1890). The principles of psychology. New York: Holt.

Jansson, E., Wilson, A. D., Williams, J. H., \& Mon-Williams, M. (2007). Methodological problems undermine tests of the ideo-motor conjecture. Experimental Brain Research, 182, 549-58.

Keysers C., \& Perrett D. I. (2004). Demystifying social cognition: a Hebbian perspective. . Trends in Cognitive Sciences, 8(11), 501-507. 
Kilner, J., Paulignan, Y., \& Blakemore, S. (2003). An Interference Effect of Observed Biological Movement on Action. Current Biology, 13 (6), 522-525.

Kornblum, S., Hasbroucq, T., \& Osman, A. (1990). Dimensional overlap: Cognitive basis of stimulus-response compatibility-A model and taxonomy. Psychological Review, 97, 253170.

Liepelt, R., von Cramon, D. Y., \& Brass, M. (2008). What is matched in direct matching? Intention attribution modulates motor priming effects. Journal of Experimental Psychology: Human Perception and Performance, 34(3), 578-591.

Longo, M. R., \& Bertenthal, B. I. (2009). Attention modulates the specificity of automatic imitation to human actors. Experimental Brain Research, 192, 739-744.

Longo, M. R., Kosobud, A., \& Bertenthal, B. I. (2008). Automatic Imitation of Biomechanically Impossible Movements: Effects of Priming Movements vs. Goals. Journal of Experimental Psychology: Human Perception and Performance, 34(2), 489-501.

Perani, D., Fazio, F., Borghese, N. A., Tettamanti, M., Ferrari, S., Decety, J., \& Gilardi, M. C. (2001). Different brain correlates for watching real and virtual hand actions. NeuroImage, 14, 749-758.

Press, C., Bird, G., Flach, R., \& Heyes, C. (2005). Robotic movement elicits automatic imitation. Cognitive Brain Research, 25(3), 632-640.

Prinz, W. (1997). Perception and Action Planning. European Journal of Cognitive Psychology, 9 (2), 129-154.

Sebanz, N., Knoblich, G., \& Prinz, W. (2003). Representing others' actions: Just like one's own? Cognition, 88, B11-B21.

Stanley J., Gowen, E. \& Miall R. C. (2007). Effects of Agency on Movement Interference During Observation of a Moving Dot Stimulus. Journal of Experimental Psychology: Human Perception and Performance, 33(4), 915-926.

Stürmer, B., Ascherschleben, G., \& Prinz, W. (2000). Correspondence effects with manual gestures and postures: a study of imitation. Journal of Experimental Psychology: Human Perception and Performance, 26, 1746-1759.

Tai, Y. F., Scherfler, C., Brooks, D. J., Sawamoto, N., Castiello, U. (2004). The human premotor cortex is 'mirror' only for biological actions. Current Biology 14, 117-120. 
Tsai, C. C., \& Brass, M. (2007). Does the human motor system simulate Pinocchio's actions? Co-acting with a human hand versus a wooden hand in a dyadic interaction. Psychological Science, 18, 1058-1062.

Tsai, C. C., Kuo, W. J., Hung, D., \& Tzeng, O. (2008). Action Co-representation is Tuned to Other Humans. Journal of Cognitive Neuroscience. 


\section{Figure captions}

Figure 1. The figures shows illustrations of (A) a human-hand model, (B) a wooden-hand model used to manipulate participants belief and (C) the stimulus display, a leather-hand movement in a congruent condition of the present experiment.

Figure 2. Shown is the stimulus sequence for each trial, depicting a finger movement as used in the present experiment. Each trial started with a picture (displayed for $800 \mathrm{~ms}$ ) showing a static hand. In the second frame (displayed for $1915 \mathrm{~ms}$ ) the symbolic imperative stimulus ' 1 ' or ' 2 ' appeared between a lifted index or middle finger. The symbolic stimulus and the moving finger appeared together. Participants had to respond within 2000 ms. The reaction was followed by a tonal feedback for $50 \mathrm{~ms}$ and a constant inter-stimulus interval (ISI).

Figure 3a. Mean reaction times (in milliseconds) of the present experiment as a function of group (human-hand model group and wooden-hand model group) and congruency (con: congruent and icon: incongruent). Error bars represent standard deviations of the mean. Figure 3b. Mean errors (in percent) of the present experiment as a function of group (humanhand model group and wooden-hand model group) and congruency (con: congruent and icon: incongruent). Error bars represent standard deviations of the mean.

Figure 4. Effect sizes of the congruency effects (in milliseconds) of the present experiment as a function of group (human-hand model group and wooden-hand model group). Error bars represent standard deviations of the mean. 
Figure 1: (A) Human-hand model, (B) Wooden-hand model and (C) Stimulus display showing a leather-hand movement in a congruent condition.

A

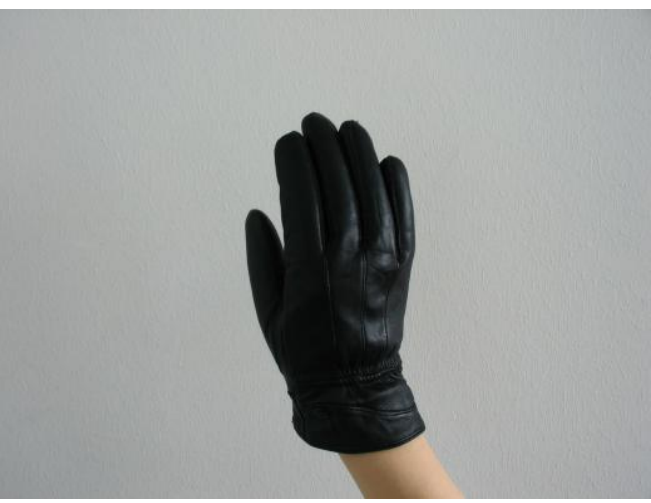

B

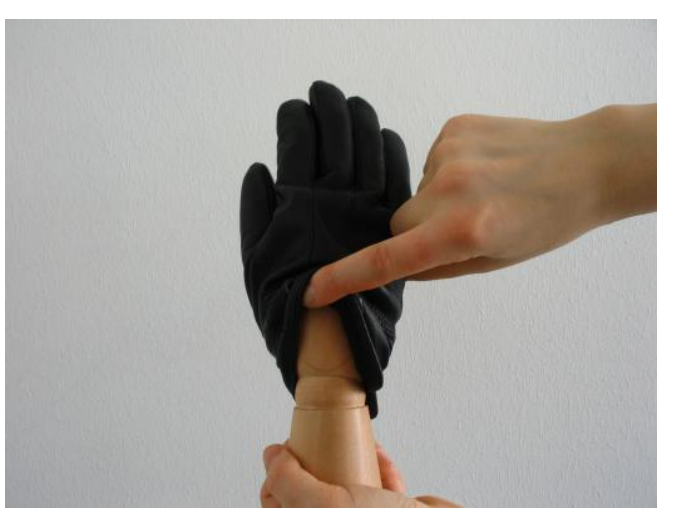

C

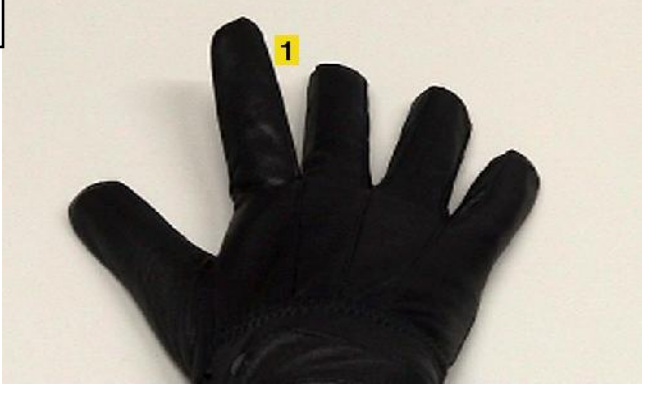


Figure 2: Trial sequence

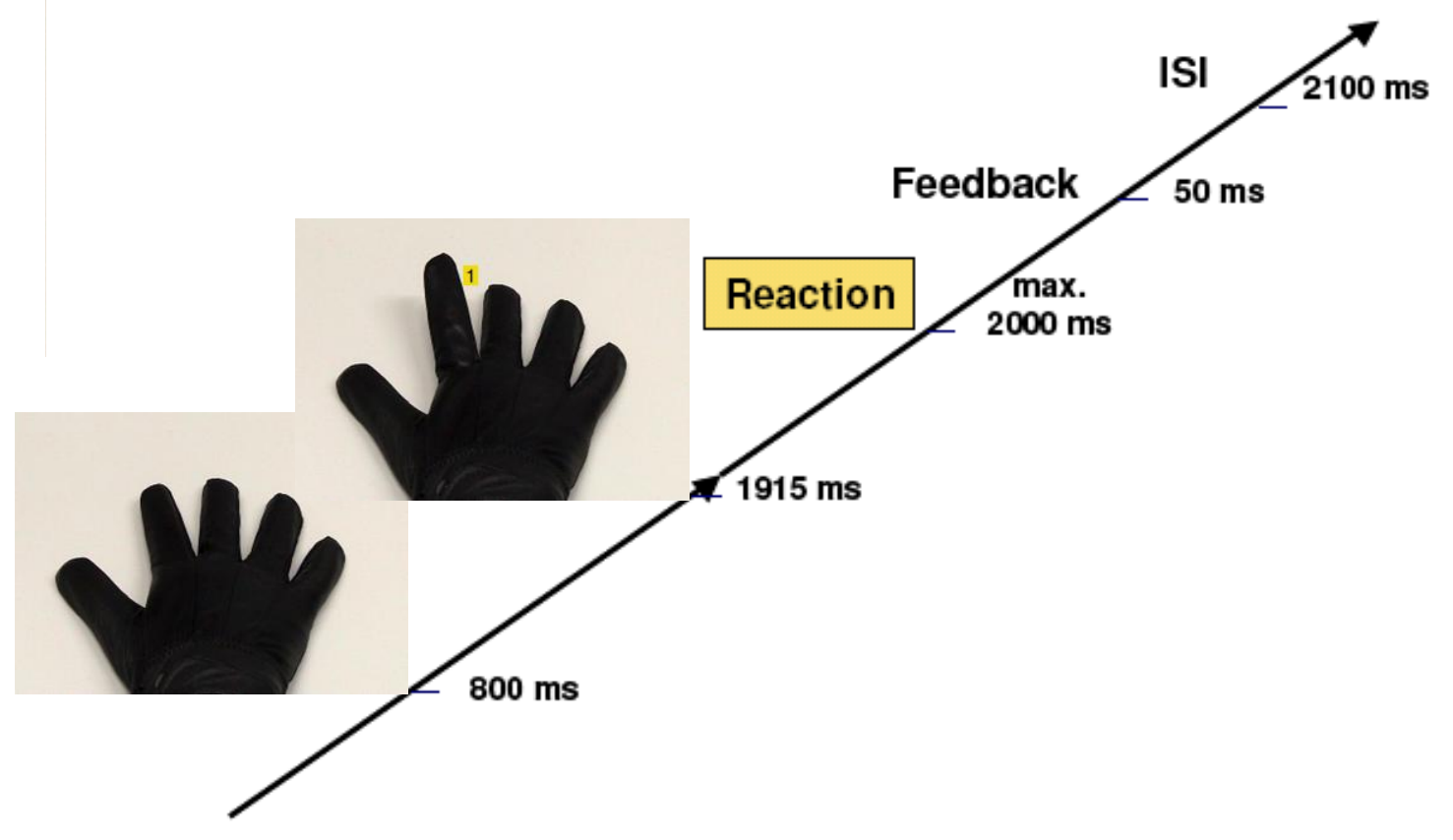


Figure 3a: Mean reaction times

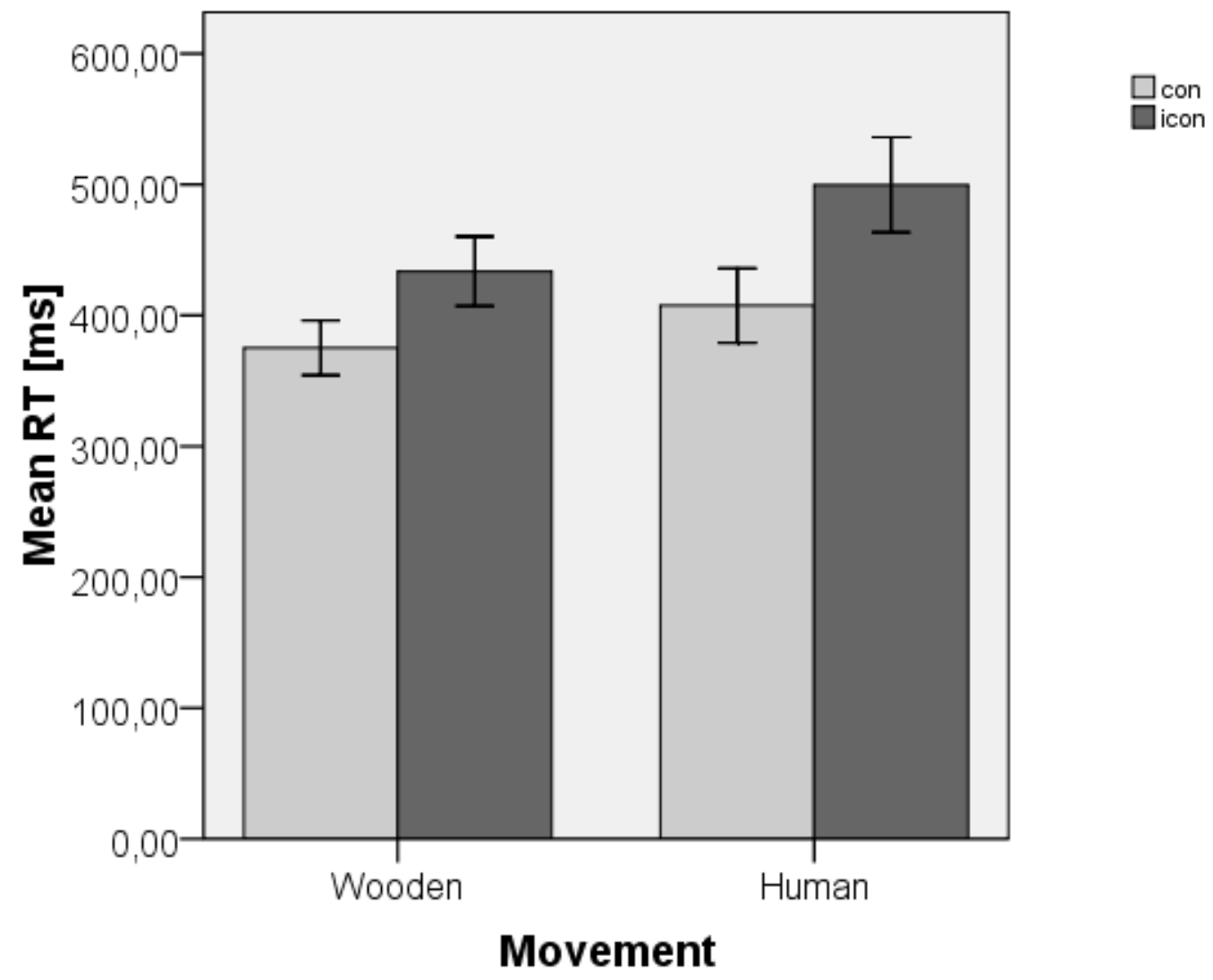


Figure 3b: Mean percent errors

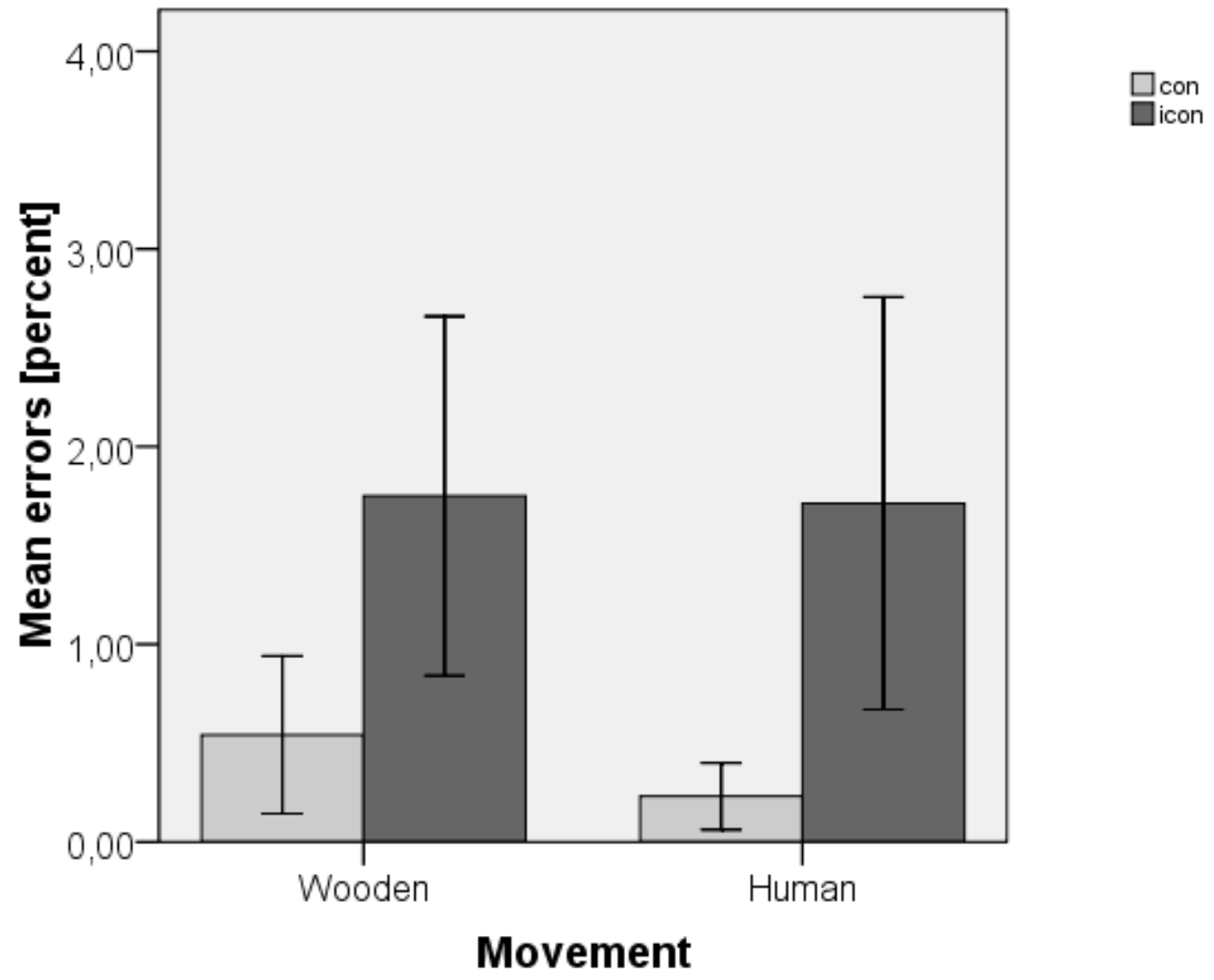


Figure 4: Effect sizes of the congruency effect.

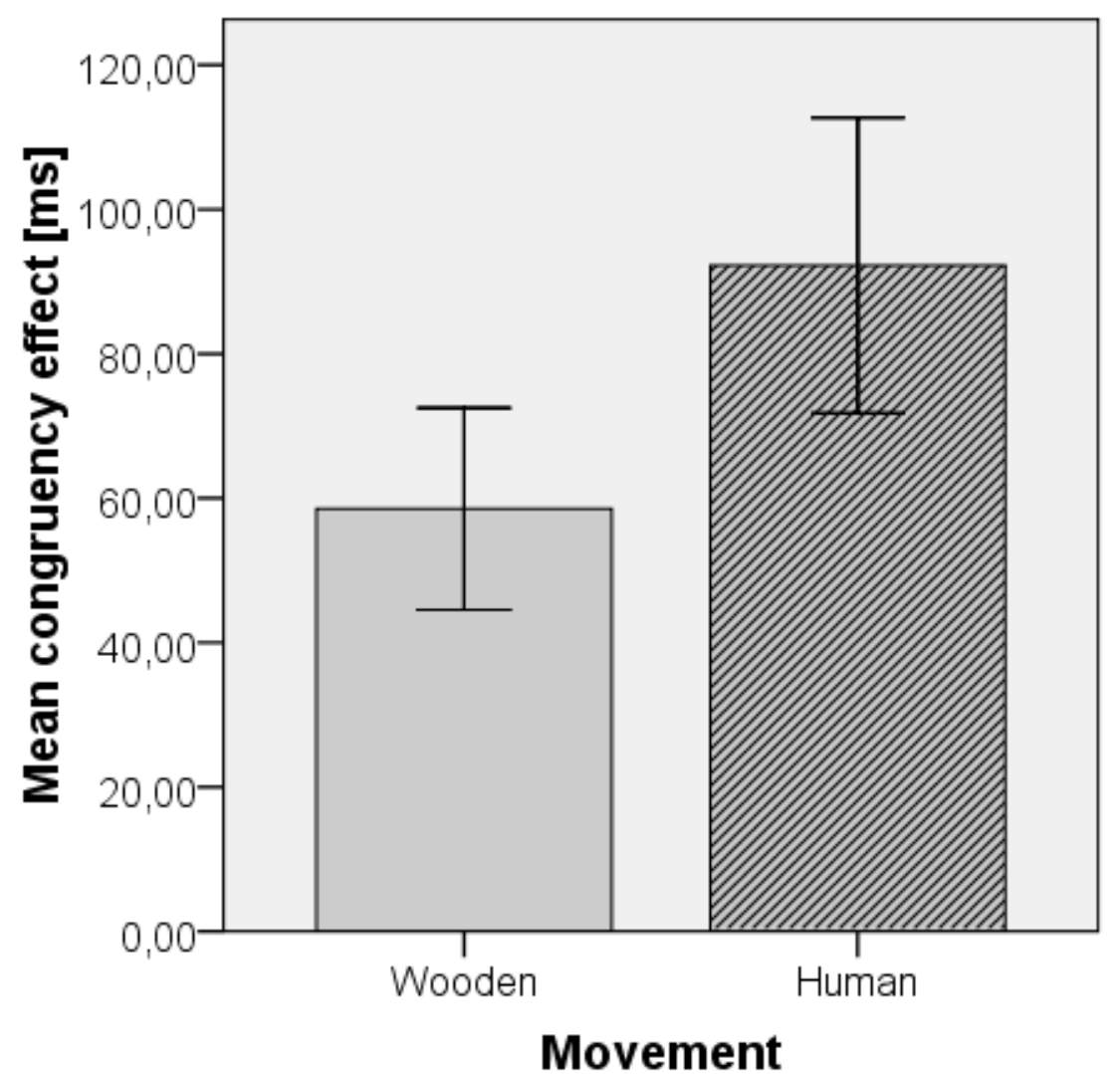


Acknowledgements:

This research was supported by a European funded project aimed at understanding the evolutionary, developmental and intentional control of imitation (EDICI-12929). The authors wish to thank Katharina Schümberg for help in data acquisition. Correspondence concerning this article should be addressed to Roman Liepelt, Max Planck Institute for Human Cognitive and Brain Sciences, Department of Psychology, Stephanstr. 1A, 04103 Leipzig, Germany. Email: liepelt@cbs.mpg.de 